\title{
Compatible Poisson-Lie structures on the loop group of $S L_{2}$.
}

\author{
B. EnRIQuez And V. Rubtsov
}

\begin{abstract}
We define a 1-parameter family of $r$-matrices on the loop algebra of $s l_{2}$, defining compatible Poisson structures on the associated loop group, which degenerate into the rational and trigonometric structures, and study the Manin triples associated to them.
\end{abstract}

Introduction. The concept of bi-(or multi-)Hamiltonian structures on manifolds is known, since the works of Magri and Gelfand-Dorfman ([Ma], [GD]), to play an important role in the study of classical integrable systems. Up to now, it still remains unclear whether this notion has any reasonable quantum analogue. Among long-known examples of such structures are the KdV hierarchy phase spaces. In that case, higher compatible Poisson structures are nonlocal, and are expected to be quantized using (nonlocal) vertex operator algebras. This program is very far from its goals at the moment.

An other example of bi-Hamiltonian manifolds comes from the theory of Poisson-Lie groups. It is an elementary fact that the rational and trigonometric structures (according to the terminology of $[\mathrm{BD}]$ ) on loop groups, are compatible. Accordingly, it is a natural question to find higher compatible Poisson structures on these groups. In this paper, we answer this question in the $s l_{2}$ case. We perform an elementary manipulation on the rational $r$-matrix, that after the operation of shifting of the spectral parameter generates the rational and trigonometric $r$-matrices. This shows the compatibility of these Poisson structures. We then study the associated Manin triples. They are of a slightly different kind from those studied in [D]. It would be interesting to achieve explicitly the quantization of the structures found here.

A possible connection between the two problems mentioned above could be the remark, that the Poisson brackets of the KdV monodromy operators in the first (resp. second) Hamiltonian structures are respectively given by the rational and trigonometric $r$-matrices $([\mathrm{FT}])$.

1. Compatible $r$-matrices for $s l_{2}\left(\mathbf{C}\left(\left(\lambda^{-1}\right)\right)\right)$.

Let $e, f, h$ be the Chevalley basis of $a=s l_{2}(\mathbf{C}), t=h \otimes h+2(e \otimes f+f \otimes e)$ the split Casimir element of $s l_{2}(\mathbf{C})$. The rational and trigonometric $r$-matrices of $a(\mathbf{C}((\lambda)))$ are

$$
r_{1}(\lambda, \mu)=\frac{t}{\lambda-\mu} \quad \text { and } \quad r_{2}(\lambda, \mu)=\frac{\lambda+\mu}{\lambda-\mu} t+2(e \otimes f+f \otimes e)
$$

They can be considered as elements of $U \operatorname{sl}_{2}(\mathbf{C})^{\otimes 2} \otimes \mathbf{C}(\lambda, \mu)$, and satisfy the Yang-Baxter equation with parameters, that is

$$
\llbracket r, r \rrbracket(\lambda, \mu, \nu):=\left[r^{12}(\lambda, \mu), r^{13}(\lambda, \nu)\right]+\text { cyclic permutations }=0,
$$


in the algebra $U s l_{2}(\mathbf{C})^{\otimes 3} \otimes \mathbf{C}(\lambda, \mu, \nu)$. Let us perform the following transformation on $r_{1}$ : we replace $\lambda$ and $\mu$ by their inverses and apply the affine Weyl group transformation $\lambda^{k} e \rightarrow \lambda^{k+1} e, \lambda^{k} h \rightarrow \lambda^{k} h, \lambda^{k} f \rightarrow \lambda^{k-1} f$. Up to sign the resulting $r$-matrix is

$$
r_{3}(\lambda, \mu)=\frac{\lambda \mu}{\lambda-\mu} h \otimes h+2 \frac{\lambda^{2}}{\lambda-\mu} e \otimes f+2 \frac{\mu^{2}}{\lambda-\mu} f \otimes e=\frac{\lambda \mu}{\lambda-\mu} t+2 \lambda e \otimes f-2 \mu f \otimes e
$$

Adding a quantity $E$ to $\lambda$ and $\mu$ transforms $r_{3}$ into $r_{3}+E r_{2}+E^{2} r_{1}$, so $\llbracket r_{3}+E r_{2}+$ $E^{2} r_{1}, r_{3}+E r_{2}+E^{2} r_{1} \rrbracket=0$. From this follows that $\llbracket r_{1}, r_{2} \rrbracket=\llbracket r_{2}, r_{3} \rrbracket=0$ and also that $\llbracket r_{1}, r_{3} \rrbracket=0$ (since we know that $\llbracket r_{2}, r_{2} \rrbracket=0$; we set $\llbracket a, b \rrbracket=\left[a^{12}(\lambda, \mu), b^{13}(\lambda, \nu)\right]+$ $\left[b^{12}(\lambda, \mu), a^{13}(\lambda, \nu)\right]+$ c.p. $)$. Hence, the Poisson structures defined on $S L_{2}(\mathbf{C}((\lambda)))$ by $r_{1}$, $r_{2}$ and $r_{3}$ are compatible (in the sense of [Ma], [GD]). Let $\tau_{E}^{(0)}$ be the automorphism of $\mathbf{C}\left(\left(\lambda^{-1}\right)\right)$ defined by $\tau_{E}\left(\lambda^{k}\right)=(\lambda+E)^{k}=\lambda^{k}+k E \lambda^{k-1}+\ldots$ and let us call $\tau_{E}$ the automorphism induced on $S L_{2}\left(\mathbf{C}\left(\left(\lambda^{-1}\right)\right)\right)$. We have:

Proposition. For $a_{i}$ complex numbers $(i=1,2,3)$, the multiplicative bivectors defined by $\sum_{i=1}^{3} a_{i} r_{i}$ define structures of Poisson-Lie group on $S L_{2}\left(\mathbf{C}\left(\left(\lambda^{-1}\right)\right)\right)$. Up to multiplication and the action of the automorphisms $\tau_{E}$, this family is reduced to a 1-parameter family, with special points the rational and trigonometric structures.

\section{Modified Yang-Baxter equation.}

Let us remark that solutions to the Yang-Baxter equation with parameters usually correspond to solutions of the modified Yang-Baxter equation, after interpretation of $r$-matrices as elements of $\left[s l_{2}\left(\mathbf{C}\left(\left(\lambda^{-1}\right)\right)\right) \otimes s l_{2}\left(\mathbf{C}\left(\left(\mu^{-1}\right)\right)\right)\right]^{-}\left({ }^{-}\right.$means direct product of homogeneous components, where the grading is $\operatorname{deg}\left(x \lambda^{k} \otimes 1\right)=\operatorname{deg}\left(1 \otimes x \mu^{k}\right)=k$ if $x \in s l_{2}(\mathbf{C})$ and $k \in \mathbf{Z}$, and $\operatorname{deg} \lambda=\operatorname{deg} \mu=1)$. Indeed, by this interpretation the $r$-matrices $r_{i}, i=1,2,3$ correspond respectively to

$$
\begin{gathered}
\bar{r}_{1}=\frac{1}{2}\left(\frac{1}{\lambda-\mu}-\frac{1}{\mu-\lambda}\right) t, \bar{r}_{2}=\frac{1}{2}\left(\frac{\lambda+\mu}{\lambda-\mu}-\frac{\lambda+\mu}{\mu-\lambda}\right) t+2(e \otimes f-f \otimes e), \\
\bar{r}_{3}=\frac{1}{2}\left(\frac{\lambda \mu}{\lambda-\mu}-\frac{\lambda \mu}{\mu-\lambda}\right) t+2 \lambda e \otimes f-2 \mu f \otimes e
\end{gathered}
$$

(we use the convention $\frac{1}{\lambda-\mu}=\sum_{i \geq 0} \mu^{i} / \lambda^{i+1}$ ).

Let us now consider the tensors

$$
t_{1}=\frac{1}{2}\left(\frac{1}{\lambda-\mu}+\frac{1}{\mu-\lambda}\right) t, t_{2}=\frac{1}{2}\left(\frac{\lambda+\mu}{\lambda-\mu}+\frac{\lambda+\mu}{\mu-\lambda}\right) t, t_{3}=\frac{1}{2}\left(\frac{\lambda \mu}{\lambda-\mu}+\frac{\lambda \mu}{\mu-\lambda}\right) t
$$

They can be considered as the split Casimir elements corresponding to the invariant pairings on $s l_{2}\left(\mathbf{C}\left(\left(\lambda^{-1}\right)\right)\right)$

$$
\begin{gathered}
\langle a(\lambda), b(\lambda)\rangle_{1}=\operatorname{res}_{\lambda=0}\langle a(\lambda), b(\lambda)\rangle d \lambda,\langle a(\lambda), b(\lambda)\rangle_{2}=\frac{1}{2} \operatorname{res}_{\lambda=0}\langle a(\lambda), b(\lambda)\rangle \frac{d \lambda}{\lambda}, \\
\langle a(\lambda), b(\lambda)\rangle_{3}=\operatorname{res}_{\lambda=0}\langle a(\lambda), b(\lambda)\rangle \frac{d \lambda}{\lambda^{2}}
\end{gathered}
$$

which allow the interpretation of $\left(s l_{2}\left(\mathbf{C}\left(\left(\lambda^{-1}\right)\right)\right), \bar{r}_{i}\right)$ as double algebras (see [D]); here $\langle$, is the Killing form on $s_{2}(\mathbf{C})$. 
Lemma. Let $a_{i}$ be numbers $(i=1,2,3)$, then we have

$$
\llbracket \sum_{i} a_{i} \bar{r}_{i}, \sum_{i} a_{i} \bar{r}_{i} \rrbracket=\llbracket \sum_{i} a_{i} t_{i}, \sum_{i} a_{i} t_{i} \rrbracket .
$$

Proof. We first show (3) for $a_{1}=a_{2}=0$ : in this case, it follows from the structure of the Manin triple in the rational case; we can also see it as a consequence of the identity (in formal series) $\frac{\lambda \mu}{\lambda-\mu} \frac{\mu \nu}{\mu-\nu}+$ p.c. $=0$ (itself a consequence of $\frac{1}{\mu^{-1}-\lambda^{-1}} \frac{1}{\nu^{-1}-\mu^{-1}}+$ p.c. $=0$ ). We prove (3) for $a_{1}=a_{3}=0$ and $a_{2}=a_{3}=0$ similarly. Returning now to the identity for $a_{2}=a_{3}=0$, the action of $\tau_{E}$ transforms $t_{3}$ into $t_{3}+E t_{2}+E^{2} t_{1}$, and $\bar{r}_{3}$ into $\bar{r}_{3}+E \bar{r}_{2}+E^{2} \bar{r}_{1}$, so we have $\llbracket \bar{r}_{3}+E \bar{r}_{2}+E^{2} \bar{r}_{1}, \bar{r}_{3}+E \bar{r}_{2}+E^{2} \bar{r}_{1} \rrbracket=\llbracket t_{3}+E t_{2}+E^{2} t_{1}, t_{3}+E t_{2}+E^{2} t_{1} \rrbracket$; so we deduce from $\llbracket \bar{r}_{i}, \bar{r}_{j} \rrbracket=\llbracket t_{i}, t_{j} \rrbracket$ for $i, j \leq 2$ or $i, j \geq 2$, the same identity for all $i, j$.

\section{Manin triples.}

Let us now consider $g=s l_{2}\left(\mathbf{C}\left(\left(\lambda^{-1}\right)\right)\right)$, endowed with the Poisson-Lie structure given by $\sum a_{i} r_{i}$. Our aim in this section is to describe it as a double algebra. For this, and following [STS], we study the eigenvalues of the operator $R: g \rightarrow g$ defined by $R A=$ $\left\langle\sum_{i} a_{i} r_{i}, A \otimes 1\right\rangle_{\left(a_{i}\right)}$, where $\langle,\rangle_{\left(a_{i}\right)}$ is the scalar product corresponding to $\sum a_{i} t_{i}$

Let us compute $\langle,\rangle_{\left(a_{i}\right)} \cdot \sum_{i} a_{i} t_{i}=\sum a_{i+j+2}^{\prime}\left(2 e \lambda^{i} \otimes f \mu^{j}+2 f \lambda^{i} \otimes e \lambda^{j}+h \lambda^{i} \otimes h \mu^{j}\right)$, with $a_{1,3}^{\prime}=a_{1,3}$ and $a_{2}^{\prime}=2 a_{2}$. The inverse of the matrix $A$ with coefficients $A_{i j}=a_{i+j+2}$ has coefficients $\left(A^{-1}\right)_{i j}=b_{i+j}$, where $b_{n}$ is the coefficient of $\lambda^{-n}$ in the expansion of $\left(a_{1} \lambda^{-1}+a_{2}+a_{3} \lambda\right)^{-1}$. We will have $\left\langle e \lambda^{i}, f \mu^{j}\right\rangle_{\left(a_{i}\right)}=\frac{1}{2}\left\langle h \lambda^{i}, h \mu^{j}\right\rangle_{\left(a_{i}\right)}=b_{i+j}$, and so

$$
\langle A(\lambda), B(\lambda)\rangle_{\left(a_{i}\right)}=\operatorname{res}_{\lambda=\infty}\langle A(\lambda), B(\lambda)\rangle \frac{d \lambda}{a_{1}+2 a_{2} \lambda+a_{3} \lambda^{2}}
$$

We can then express $R$ as

$$
\begin{aligned}
R(A)(\mu) & =\langle r(\lambda, \mu), A(\lambda)\rangle_{\left(a_{i}\right)} \\
& =\operatorname{res}_{\lambda=\infty}\left\{t\left(a_{1}+(\lambda+\mu) a_{2}+\lambda \mu a_{3}\right) \frac{1}{2}\left(\frac{1}{\lambda-\mu}-\frac{1}{\mu-\lambda}\right)+2\left(a_{2}+a_{3} \lambda\right) e \otimes f\right. \\
& \left.-2\left(a_{2}+a_{3} \mu\right) f \otimes e\right\} A(\lambda) \frac{d \lambda}{a_{1}+2 a_{2} \lambda+a_{3} \lambda^{2}} \\
& =\operatorname{res}_{\lambda=\infty} t A(\lambda) \frac{1}{2}\left(\frac{1}{\lambda-\mu}-\frac{1}{\mu-\lambda}\right) d \lambda+\operatorname{res}_{\lambda=\infty} \frac{-t\left(a_{2}+a_{3} \lambda\right)}{a_{1}+2 a_{2} \lambda+a_{3} \lambda^{2}} A(\lambda) d \lambda \\
& +\operatorname{res}_{\lambda=\infty} \frac{2 e \otimes f\left(a_{2}+a_{3} \lambda\right)}{a_{1}+2 a_{2} \lambda+a_{3} \lambda^{2}} A(\lambda) d \lambda-\operatorname{res}_{\lambda=\infty} \frac{2 f \otimes e\left(a_{2}+a_{3} \mu\right)}{a_{1}+2 a_{2} \lambda+a_{3} \lambda^{2}} A(\lambda) d \lambda,
\end{aligned}
$$

where $\langle$,$\rangle is understood in the two last lines. Since \langle t, A\rangle=2 A(\langle a, b\rangle$ is the trace of $a b$ in the fundamental representation), and denoting for a formal series $\phi=\sum \phi_{i} \lambda^{i}, \phi_{>0}=$ $\sum_{i>0} \phi_{i} \lambda^{i}$ and $\phi_{\leq 0}=\sum_{i \leq 0} \phi_{i} \lambda^{i}$, we get for the first term $A(\mu)_{>0}-A(\mu)_{\leq 0}$.

We see that $R$ acts as 1 on $a \otimes \mathbf{C}\left[\left[\lambda^{-1}\right]\right]$, and -1 on $f \otimes \lambda \mathbf{C}[\lambda]$. In addition, the subspace $h \otimes \mathbf{C}[\lambda] \oplus e \otimes \lambda^{-1} \mathbf{C}[\lambda]$ is stable under $R$. Let $A=\sum_{i \geq 0} \alpha_{i} h \lambda^{i}+\sum_{i \geq-1} \beta_{i} e \lambda^{i}$ belong to this space. We now restrict ourselves to the particular case $a_{1}=1, a_{2}=0$ and $a_{3}=-1$. Pose $R A=\sum_{i \leq 0} \alpha_{i}^{\prime} h \lambda^{i}+\sum_{i \leq 1} \beta_{i}^{\prime} e \lambda^{i}$. Then

$$
\alpha_{0}^{\prime}=\alpha_{0}+2\left(\alpha_{2}+\alpha_{4}+\cdots\right), \beta_{0}^{\prime}=\beta_{0}+2\left(\beta_{2}+\beta_{4}+\cdots\right), \beta_{1}^{\prime}=\beta_{-1}+2\left(\beta_{1}+\beta_{3}+\cdots\right),
$$


and

$$
\alpha_{i}^{\prime}=-\alpha_{i}, \quad \beta_{i}^{\prime}=-\beta_{i} \text { for } i<0 .
$$

The space is then the sum of the eigenspaces $\mathbf{C} h \oplus \mathbf{C} e \oplus \mathbf{C} e \lambda^{-1}$ for the eigenvalue 1, and $\left\{A(\lambda) \mid \alpha_{0}+\alpha_{2}+\cdots=0, \beta_{0}+\beta_{2}+\cdots=0, \beta_{-1}+\beta_{1}+\beta_{3} \cdots=0\right\}$ for -1 . So we have:

Proposition. The eigenspaces of $R$ are $a\left[\left[\lambda^{-1}\right]\right]$ and $g_{+}$, where $g_{+}$is the subspace of $a \otimes \mathbf{C}(\lambda)$ of functions $A(\lambda)$ with only poles zero and infinity, and such that $A(1)$ and $A(-1) \in b_{-},[A(1)+A(-1)]_{h}=0$, and $A \in \frac{1}{\lambda} n_{+} \oplus b_{+} \oplus \lambda a[[\lambda]]$ (in the completion at 0 $a \otimes \mathbf{C}((\lambda)))$. Here $n_{+}=\mathbf{C} e, \underline{h}=\mathbf{C} h, b_{+}=\underline{h} \oplus n_{+}, b_{-}=\mathbf{C} h+\mathbf{C} f,[]_{h}$ is the natural projection from $b_{-}$to $\underline{h}$.

These two spaces are supplementary Lie subalgebras of $g$, isotropic for the form

$$
\langle A(\lambda), B(\lambda)\rangle_{(1,0,-1)}=\operatorname{res}_{\lambda=\infty}\langle A(\lambda), B(\lambda)\rangle \frac{d \lambda}{1-\lambda^{2}}
$$

So the decomposition $g=g_{+} \oplus a[\lambda]$, together with the above scalar product on $g$, defines a Manin triple.

These last facts can be checked directly, for example for the isotropy of $g_{+}$, the residue formula gives: if $A$ and $B \in g_{+}$, then

$$
\begin{aligned}
\langle A(\lambda), B(\lambda)\rangle_{\left(a_{i}\right)} & =-\operatorname{res}_{\lambda=1}\langle A, B\rangle \frac{d \lambda}{\lambda-1} \frac{-1}{\lambda+1}-\operatorname{res}_{\lambda=-1}\langle A, B\rangle \frac{d \lambda}{\lambda+1} \frac{-1}{\lambda-1} \\
& -\operatorname{res}_{\lambda=0}\langle A, B\rangle \frac{d \lambda}{1-\lambda^{2}} \\
& =\frac{1}{2}\left(\operatorname{res}_{\lambda=1}\langle A, B\rangle \frac{d \lambda}{\lambda-1}-\operatorname{res}_{\lambda=-1}\langle A, B\rangle \frac{d \lambda}{\lambda+1}\right) \\
& -\operatorname{res}_{\lambda=0}\left\langle\frac{1}{\lambda} n_{+}^{A}+b_{+}^{A}+\cdots, \frac{1}{\lambda} n_{+}^{B}+b_{+}^{B}+\cdots\right\rangle d \lambda\left(1+\lambda^{2}+\cdots\right)
\end{aligned}
$$

The first term is zero by the compatibility conditions at points 1 and -1 , and the second vanishes also.

Remarks.

1. Remind, that a solution $r(\lambda, \mu)$ of CYBE is called non-degenerate if it is nondegenerate for at least one point $(\lambda, \mu)$. Drinfeld conjectered that every non-degenerate rational $r$-matrix $r(\lambda, \mu)$ is equivalent to a solution of CYBE of the form

$$
r(\lambda, \mu)=\frac{t}{\lambda-\mu}+P(\lambda, \mu)
$$

where $P(\lambda, \mu)$ is a polynomial of degree at most one in each variables. This problem was investigated by A. Stolin in $[\mathrm{S}]$ and it follows from his results that in the $\operatorname{sl}_{2}(\mathbf{C})$-case there are two rational $r$-matrices:

$$
r_{1}^{\prime}(\lambda, \mu)=\frac{t}{\lambda-\mu}+2(h \otimes e-e \otimes h)
$$




$$
r_{1}^{\prime \prime}(\lambda, \mu)=\frac{t}{\lambda-\mu}+2(\mu h \otimes e-\lambda e \otimes h)
$$

We can perform the manipulations of sect. 1 on the above matrices. Starting with the $r$-matrices (5) and (6), we obtain the $r$-matrices

$$
r_{3}^{\prime}(\lambda, \mu)=\frac{\lambda \mu t}{\lambda-\mu}+2(\lambda e \otimes f+\mu f \otimes e)+2(\mu h \otimes e-\lambda e \otimes h)
$$

and

$$
r_{3}^{\prime \prime}(\lambda, \mu)=\frac{\lambda \mu t}{\lambda-\mu}+2(\lambda e \otimes f+\mu f \otimes e)+2(h \otimes e-e \otimes h),
$$

which can be included in the similar compatible triple of multiplicative Poisson structures (the bialgebra structure corresponding to $r_{1}^{\prime}$ has been quantized recently in [KhST]).

2. In the case of different parameters $\left(a_{i}\right)$, the points 1 and -1 should be shifted to some other points $z_{i}$ of $\mathbf{C}^{*}$ (in the same time the form $\frac{d \lambda}{\lambda^{2}-1}$ is changed to $\left.\frac{d \lambda}{\left(\lambda-z_{1}\right)\left(\lambda-z_{2}\right)}\right)$. It would be interesting to see how this family a Manin triples degenerates to the known cases rational and trigonometric ones (as they are described in [D]).

3. It could also be interesting to generalize this to a situation with more marked points. We see that the description given here does not fit in the adelic framework if [D] (the form is not the sum of residues at all singular points but only the residue at one point, and the "evident" half of Manin pair is not the algebra of functions regular outside these points).

Acknowledgements. The authors express their gratitude to V. Fock for discussions related to the matter of this text, and to A.Yu. Orlov for discussions concerning the Poisson brackets of monodromy operators in the higher Hamiltonian structures.

This paper was partly written during the visit paid by the second author to the Ecole Polytechnique, to whom he would like to express his thanks. The second author is supported by ISF grants MGK000, and RFFI 94-02-03379 and 94-02-14365, and also supported by the CNRS.

\section{REFERENCES}

[BD] A.A. Belavin, V.G. Drinfeld, Solutions of the classical Yang-Baxter equation for simple Lie algebras, Funct. An. Appl. 16:3 (1981), 159-80.

[D] V.G. Drinfeld, Quantum groups, Proc. ICM-86 1 (1987), 798-820.

[FT] L.D. Faddeev, L.A. Takhtajan, Hamiltonian methods in soliton equations, Springer-Verlag, Berlin, 1992.

[GD] I.M. Gelfand, I.Ya. Dorfman, Schouten brackets and Hamiltonian operators, Funct. An. Appl. 14:3 (1980), 71-4.

[KhST] S.M. Khoroshkin, A.A. Stolin, V.N. Tolstoy, Deformation of Yangian Y(sl 2$)$, q-alg/9511005 (1995).

[M] F. Magri, A geometric approach to the nonlinear solvable equations, Lect. Notes in Physics 120 (1980), Springer, 233-63.

[STS] M.A. Semenov-Tian-Shansky, What is a classical r-matrix?, Funct. An. Appl. 17 (1983), $259-72$.

[S] A.Stolin, On rational solutions of Yang-Baxter equations. Maximal orders in loop algebras, Commun. Math. Phys. 141(3) (1991), 533-48.

B.E., V.R.: Centre de Mathématiques, Ecole Polytechnique, URA 169 du CNRS, F-91128 Palaiseau, France

V.R.(Permanent address): ITEP, B. Cheremushininskaya, 117259, Moscou, Russie 